\title{
Scattering Attenuation of SH-Waves in Fiber-Reinforced Composites with Partial Imperfectly Bonded Interfaces
}

\author{
Jun Zhang $\mathbb{D}^{1},{ }^{1,2}$ Longhai Zeng, ${ }^{1}$ and Lian Liu ${ }^{1}$ \\ ${ }^{1}$ College of Aerospace Engineering, Chongqing University, Chongqing 400044, China \\ ${ }^{2}$ Chongqing Key Laboratory of Heterogeneous Material Mechanics, Chongqing University, Chongqing 400044, China \\ Correspondence should be addressed to Jun Zhang; mejzhang@cqu.edu.cn
}

Received 28 October 2017; Accepted 1 January 2018; Published 20 February 2018

Academic Editor: Nerio Tullini

Copyright ( 2018 Jun Zhang et al. This is an open access article distributed under the Creative Commons Attribution License, which permits unrestricted use, distribution, and reproduction in any medium, provided the original work is properly cited.

\begin{abstract}
The influence of partial imperfectly bonded interfaces between each fiber and the matrix on the scattering attenuation of coherent $\mathrm{SH}$-waves in fiber-reinforced composites is investigated. The imperfection of interfaces is modelled using the spring model, in which the level of imperfection is characterized by a parameter $K$, which is called the stiffness of the imperfect interfaces. First, the single scattering of $\mathrm{SH}$-waves by a cylindrical fiber with such a partial imperfectly bonded interface to the matrix is formulated and subsequently solved using the collocation point (CP) method. Later on, based on the analysis of the corresponding single scattering problem, effects of several parameters (i.e., frequency of the incident wave, level of imperfection of interfaces, and width of the imperfect interfaces) on the far-field scattering magnitude, scattering cross section, and scattering attenuation coefficients of coherent $\mathrm{SH}$-waves are shown graphically. The potential application of the current results to nondestructive evaluation of interfaces in composites is also discussed.
\end{abstract}

\section{Introduction}

Various interfaces exist in the nature, such as interfaces between different constituents of composite materials and those between distinct phases in a polycrystalline material. For composite materials, the interfaces play very important roles, such as transferring loads from the matrix to fibers. Accordingly, evaluation of the interfaces of composite materials has been the focus of many researchers [1-6].

Currently, the ultrasonic technique has become a major method for the nondestructive evaluation of interfaces, due to the fact that the behavior (i.e., attenuation and wave speed) of elastic waves in composite materials is highly related to the interfaces $[1,2,7]$. It is well known that, before using this ultrasonic technique to evaluate composite materials, the relationship between characteristics of elastic waves in composites and their properties should be established. It is in this spirit that, to date, numerous theoretical models [8-20] for the evaluation of attenuation and wave speed of coherent elastic waves in composite materials have been proposed. It is worthwhile to note that, among these models, there is a simple one used for cases with dilute concentration of inclusions, which is called the independent scattering model $[10,17-19,21]$. The model indicates that the scattering attenuation coefficient of coherent elastic waves in composites can be calculated as $(1 / 2) \sigma_{s} n_{0}$, where $\sigma_{s}$ is the scattering cross section [22] and $n_{0}$ is the number of fibers/particulates inside a unit area/volume. Several experiments $[1,9]$ have shown that this independent scattering model has a good performance.

To date, in most of published relevant works, the fibers and the surrounding matrix are usually assumed to be perfectly bonded. However, this is not always true in practice. Therefore, during the past few years, several theoretical models have been developed to model the imperfect situations of interfaces. These models include, but are not limited to, the spring model [23] and the spring-mass model [24]. Specifically, in the spring model, the stress is assumed to be continuous across the interfaces, while allowing the displacement has a jump across the interfaces. This jump is assumed to be proportional to the stresses at the interfaces and has the relation $\left.\sigma\right|_{r=a}=K[u]$, where $[u]$ denotes the jump of the displacements across the interface and $K$ is the parameter to characterize the imperfection level of the interfaces. It is clear 
that, when $K \rightarrow \infty$, the spring model becomes the familiar perfect bonding and it is degenerated to the debonding case as $K \rightarrow 0$. Other intermediate values of $K$ represent the "imperfect bonding" between the "perfect bonding" and the "debonding." The validity of this spring model has been verified in several works $[1,7,25]$. Compared with the spring model, the additional inertia effect of the interphases has been considered in the spring-mass model. Detailed information on these models has been provided in [26]. This spring model can be also used to model the imperfect interfaces in other physical fields, that is, electric and magnetic [27].

In the past few years, this imperfect bonding effect on the effective properties and the behavior of elastic waves in composite materials/structures have attracted attention of several researchers [1, 28-31]. Most of these works have accounted for the imperfect bonding effect through the spring model. Specifically, for example, the influence of the imperfect bonding between inclusions and the matrix on the effective elastic properties $[28,31,32]$ and thermal [33] properties of composites has been investigated. For the behavior of elastic waves in composites, Rokhlin et al. [1] have investigated the imperfect bonding effect on the scattering of $\mathrm{SH}-, \mathrm{P}-$, and $\mathrm{SV}$-waves in fiber-reinforced composites. Mal and Bose [30] have studied the phase velocity and attenuation of coherent $\mathrm{P}$ - and $\mathrm{SV}$-waves in particulate composites taking the imperfect bonding effect into account. It should be pointed out that, in most of these published works, the whole interface between each fiber/particulate and the matrix is usually assumed to enter the imperfect bonding situation simultaneously. However, this is not always the case. Assuming that the interface between each fiber and the matrix deteriorates as a process would be more reasonable. In this regard, Lopez-Realpozo et al. [31] have investigated the nonuniform imperfect bonding effect on the effective shear stiffness of a periodic fibrous composite. The corresponding work on the effective thermal conductivity has been conducted in [33]. Several other authors [3, 6, 34-38] have investigated the influence of partial debonding of interfaces on elastic wave propagation in composite materials, which is an extreme case of the partial imperfect bonding. To the best of our knowledge, the investigation of the influence of the partial imperfect bonding of interfaces on the behavior of coherent elastic waves in composites is still scarce.

In this work, we aimed to investigate the influence of the partial imperfect bonding of interfaces on the scattering attenuation of coherent $\mathrm{SH}$-waves in fiber-reinforced composite materials. The imperfection of interfaces is modelled using the spring model. The scattering attenuation is calculated using the previously introduced independent scattering model. The $\mathrm{CP}$ method is proposed to solve the corresponding single scattering problem with a partial imperfectly bonded interface. Compared with the existing techniques, the derivation process and implementation of this CP method are simple and it has a comparable accuracy. Based on this CP method, influence of various aspects of the imperfect interfaces between fibers and the matrix on the scattering attenuation of coherent $\mathrm{SH}$-waves in composites is studied. Such investigation benefits the nondestructive evaluation of the interfaces in composites using the ultrasonic

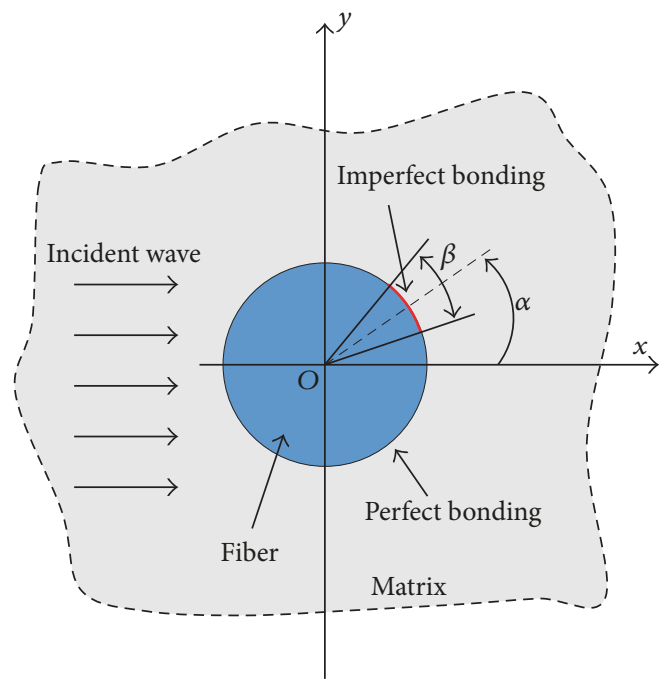

FIGURE 1: Scattering of a plane SH-wave by a cylindrical fiber with a partial imperfect bonding to the matrix. The range of the imperfect bonding is denoted as the red solid arc.

technique, as the results indicate that the working frequency of the scattering attenuation based ultrasonic technique to evaluate the interfaces should be carefully selected.

In the following sections, first, the single scattering of $\mathrm{SH}$ waves by a cylindrical fiber with a partial imperfect bonding to the matrix is described in detail. The CP method is then introduced to solve such a single scattering problem. Based on the analysis of the single scattering problem, extensive parameter analysis on the far-field scattering magnitude, scattering cross section, and scattering attenuation is then conducted. At the end, a short conclusion is presented.

\section{Single Scattering of SH-Waves by a Fiber with a Partial Imperfect Bonding to the Matrix}

2.1. Formulation of the Problem. Figure 1 shows a schematic of the single scattering of a plane $\mathrm{SH}$-wave by a cylindrical fiber with radius $a$. As shown in this figure, only a portion of the fiber is perfectly bonded to the matrix while the remaining is not. The symmetric axis of the imperfect bonding is at an angle $\alpha$ with the horizontal axis. The range of the imperfect bonding is represented by angle $\beta$, as indicated by the red solid curve in Figure 1. It is clear that, when $\beta=0$, the whole fiber is purely perfectly bonded to the matrix and the whole interface becomes a pure imperfect bonding as $\beta=2 \pi$. The incident wave is a plane SH-wave with a unit magnitude $\left(u^{\text {inc }}(x)=e^{\mathbf{i}\left(k_{m} x-\omega t\right)}\right)$ propagating in the positive horizontal direction. For harmonic analysis, the term $e^{-\mathbf{i} \omega t}$ is omitted for simplicity. In the polar coordinates, the incident wave, the wave scattered by the fiber, and the wave refracted into the fiber can be expressed as series [22] as follows:

$$
u^{\mathrm{inc}}(r, \theta)=\sum_{n=-\infty}^{\infty} \mathbf{i}^{n} J_{n}\left(k_{m} r\right) e^{\mathrm{i} n \theta}, \quad r \geq a,
$$




$$
\begin{aligned}
& u^{s}(r, \theta)=\sum_{n=-\infty}^{\infty} A_{n} H_{n}^{(1)}\left(k_{m} r\right) e^{\mathrm{i} n \theta}, \quad r \geq a, \\
& u^{f}(r, \theta)=\sum_{n=-\infty}^{\infty} B_{n} J_{n}\left(k_{f} r\right) e^{\mathrm{i} n \theta}, \quad r \leq a,
\end{aligned}
$$

where $(r, \theta)$ is the polar coordinates with origin at the center of the fiber; $k_{m}$ and $k_{f}$ are the wave numbers in the matrix and the fiber, respectively; $J_{n}(\cdot)$ and $H_{n}^{(1)}(\cdot)$ are the Bessel and Hankel function of the first kind; $\mathbf{i}=\sqrt{-1}$ is the imaginary unit; $A_{n}$ and $B_{n}$ are the unknown coefficients, which are to be determined by the conditions of displacements and stresses at the interface $(r=a)$. In this case, the conditions at the interface can be listed as follows:

$$
\begin{aligned}
& \left.\sigma_{r z}^{+}\right|_{r=a}=\left.\sigma_{r z}^{-}\right|_{r=a}, \quad \theta \in\left[\begin{array}{ll}
0 & 2 \pi
\end{array}\right] \\
& \left.u^{+}\right|_{r=a}-\left.u^{-}\right|_{r=a} \\
& = \begin{cases}\left.\frac{1}{K} \sigma_{r z}^{+}\right|_{r=a}, & \theta \in(\text { Imperfect Bonding) } \\
0, & \theta \in(\text { Perfect Bonding }),\end{cases}
\end{aligned}
$$

where $\left.\sigma_{r z}^{+}\right|_{r=a}$ and $\left.\sigma_{r z}^{-}\right|_{r=a}$ are the axial shear stresses at the interface; the superscript "+" means values in the matrix and "-" denotes those in the fiber; variable $K$ is the stiffness of the interface adopted in the spring model. Substituting of the expression of displacements in (1) and the corresponding expression for the axial shear stress into (2), the conditions at the interface described in (2) can be further expressed in detail as follows:

$$
\begin{aligned}
& \sum_{n=-\infty}^{\infty}\left[\mathbf{i}^{n} J_{n}\left(k_{m} a\right)+A_{n} H_{n}^{(1)}\left(k_{m} a\right)-\gamma \frac{\mathbf{i}^{n} J_{n}^{\prime}\left(k_{m} a\right)+A_{n} H_{n}^{(1)^{\prime}}\left(k_{m} a\right)}{J_{n}^{\prime}\left(k_{f} a\right)} J_{n}\left(k_{f} a\right)\right] e^{\mathrm{i} n \theta} \\
& \quad \begin{cases}\sum_{n=-\infty}^{\infty}\left[\mathbf{i}^{n} J_{n}^{\prime}\left(k_{m} a\right)+A_{n} H_{n}^{(1)^{\prime}}\left(k_{m} a\right)\right] \frac{\mu_{m} k_{m}}{K} e^{\mathrm{i} n \theta}, & \theta \in(\text { Imperfect Bonding) } \\
0, & \theta \in \text { (Perfect Bonding) }\end{cases}
\end{aligned}
$$

with $\gamma=\mu_{m} k_{m} / \mu_{f} k_{f}$. Here, $\mu_{m}$ and $\mu_{f}$ are the shear modulus of the matrix and the fiber. The symbol Prime means the first-order derivative. Unlike the pure perfect and imperfect bonding cases, the orthogonality of trigonometric functions has not been available to (3). Therefore, analytical solutions for $A_{n}$ and $B_{n}(n=-\infty, \ldots, \infty)$ cannot be obtained as the pure perfect and imperfect bonding cases. In this work, (3) has been solved using the CP method [39].

2.2. The CP Method to Solve (3). Previous works [39, 40] have shown that several terms of the lowest order are sufficient to obtain convergent results for the infinite series expressed in (1), for cases in which the fiber is purely perfectly bonded to the matrix. In this work, those infinite series in (1) are truncated with the maximum order $n_{\max }$. Then the number of unknown $A_{n}$ in (3) equals $2 n_{\max }+1$. In order to solve these unknowns, (3) is forced to be satisfied at $2 n_{\max }+1$ CPs. In this work, these CPs are distributed symmetrically about the partial imperfectly and perfectly bonded interfaces, respectively, as seen in Figure 2. Additionally, these CPs are required to be distributed as uniform as possible along the whole interface. Then a linear system of size $2 n_{\max }+1$ is established for $A_{n}, n=-n_{\max }, \ldots, n_{\max }$ as

$$
C_{l n} A_{n}=b_{l} \quad\left(l, n=-n_{\max }, \ldots, n_{\max }\right)
$$

with $C_{l n}=\left[H_{n}^{(1)}\left(k_{m} a\right)-\gamma\left(H_{n}^{(1)^{\prime}}\left(k_{m} a\right) / J_{n}^{\prime}\left(k_{f} a\right)\right) J_{n}\left(k_{f} a\right)-\right.$ $\left.\left(\mu_{m} k_{m} / K\right) H_{n}^{(1)^{\prime}}\left(k_{m} a\right)\right] e^{\mathbf{i} n \theta_{l}}$ and $b_{l}=\mathbf{i}^{n}\left[\left(\mu_{m} k_{m} / K\right) J_{n}^{\prime}\left(k_{m} a\right)-\right.$ $\left.J_{n}\left(k_{m} a\right)+\gamma\left(J_{n}^{\prime}\left(k_{m} a\right) / J_{n}^{\prime}\left(k_{f} a\right)\right) J_{n}\left(k_{f} a\right)\right] e^{\mathrm{i} n \theta_{l}}$ as the collocation point is located within the imperfect bonding; otherwise, $C_{l n}=\left[H_{n}^{(1)}\left(k_{m} a\right)-\gamma\left(H_{n}^{(1)^{\prime}}\left(k_{m} a\right) / J_{n}^{\prime}\left(k_{f} a\right)\right) J_{n}\left(k_{f} a\right)\right] e^{\mathrm{i} n \theta_{l}}$ and $b_{l}=-\mathbf{i}^{n} J_{n}\left(k_{m} a\right) e^{\mathbf{i} n \theta_{l}}$. The angle $\theta_{l}$ is illustrated in Figure 2. Solving (4) produces all $A_{n}$ and $B_{n}, n=-n_{\max }, \ldots, n_{\max }$. Then displacement and axial shear stress at each point in both the matrix and the fiber can be obtained.

Additionally, the two parameters, scattering cross section $\left(\sigma_{s}\right)$ [22], and the directional far-field scattering magnitude $(f(\theta))$ [20], required by those theoretical models (including the independent scattering model) to evaluate attenuation and phase velocity of coherent waves in composites, can be also calculated. $\sigma_{s}$ is defined as $\sigma_{s}=\operatorname{Ave}(\dot{E}) / \operatorname{Ave}(\dot{e})$, where the numerator is the averaged (over one period) energy flux of the scattered wave across a closed circle with a radius $R$ enclosing the fiber. It is expressed as

$$
\begin{aligned}
& \operatorname{Ave}(\dot{E}) \\
& \qquad=-\frac{\mathbf{i} \omega}{4} \int_{0}^{2 \pi}\left[\sigma_{r z}^{s} \times\left(u^{s}\right)^{*}-\left(\sigma_{r z}^{s}\right)^{*} \times u^{s}\right]_{r=R} R d \theta .
\end{aligned}
$$

Here, an asterisk indicates the complex conjugate of the corresponding quantity. Substituting the displacements of the scattered wave in (1) and the corresponding axial shear stresses into (5),

$$
\begin{aligned}
& \operatorname{Ave}(\dot{E})=-\frac{\mathbf{i} \pi \omega}{2} \mu_{m} k_{m} R \times \sum_{n=-n_{\max }}^{n_{\max }}\left|A_{n}\right|^{2} \\
& \cdot\left[H_{n}^{(1)^{\prime}}\left(k_{m} R\right) H_{n}^{(1)^{*}}\left(k_{m} R\right)\right. \\
& \left.-H_{n}^{(1)^{\prime *}}\left(k_{m} R\right) H_{n}^{(1)}\left(k_{m} R\right)\right] .
\end{aligned}
$$




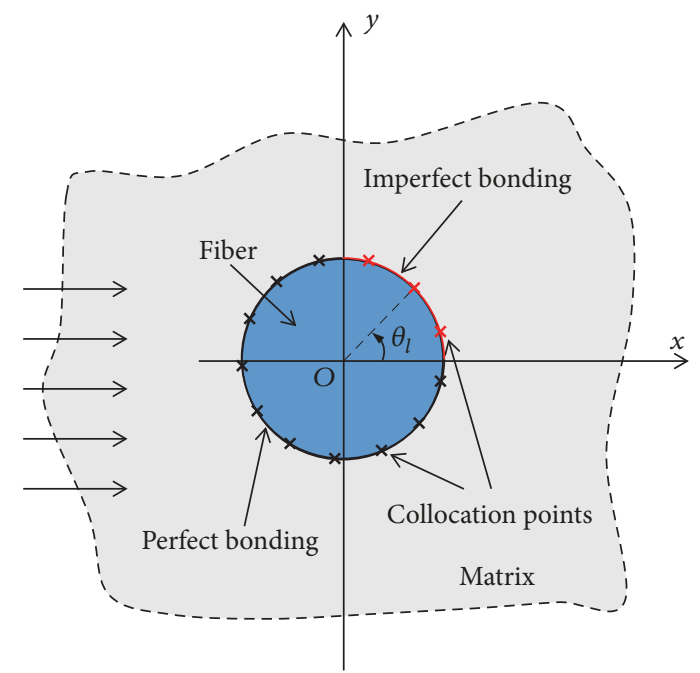

FIgURE 2: Distribution of CPs denoted by " $x$ " symbol. It is noted that at least one CP should be distributed on the imperfectly bonded interface as long as $\beta$ is not equal to 0 . In this figure, $=\pi / 4 \beta=\pi / 2$, and $n_{\max }=6$.

In the derivation of the above equation, the orthogonality of trigonometric functions has been used. As $k_{m} R \rightarrow \infty$, $H_{n}^{(1)}\left(k_{m} R\right) \rightarrow \sqrt{\left(2 / \pi k_{m} R\right)} e^{\mathbf{i}\left[k_{m} R-(n+1 / 2)(\pi / 2)\right]}$ and $H_{n}^{(1)^{\prime}}\left(k_{m} R\right)$ $\rightarrow \mathbf{i} H_{n}^{(1)}\left(k_{m} R\right)$. Therefore, the above equation becomes

$$
\operatorname{Ave}(\dot{E})=2 \omega \mu_{m} \sum_{n=-n_{\max }}^{n_{\max }}\left|A_{n}\right|^{2}
$$

as $k_{m} R \rightarrow \infty$. The denominator is the corresponding energy flux of the incident wave across a unit length, which has an expression $\operatorname{Ave}(\dot{e})=(\omega / 2) \mu_{m} k_{m}$. Then, $\sigma_{s}(\alpha, \beta)=$ $\left(4 / k_{m}\right) \sum_{n=-n_{\max }}^{n=n_{\max }}\left|A_{n}\right|^{2}$, which is a function of $\alpha$ and $\beta . \sigma_{s}$ is an evaluation of the energy scattered by the fiber compared with the incident wave, which reflects the scattering capability of the fiber.

The directional far-field scattering magnitude is defined as

$$
u^{s}(r, \theta) \approx f(\theta) \sqrt{\frac{2}{\pi k_{m} r}} e^{\mathbf{i}\left(k_{m} r-\pi / 4\right)}
$$

as $k_{m} r \rightarrow \infty$. Substituting the displacements of the scattered wave in (1), $f(\theta)$ equals $\sum_{n=-n_{\max }}^{n=n_{\max }} A_{n}(-\mathbf{i})^{n} e^{\mathbf{i} n \theta}$, which also depends on $\alpha$ and $\beta . f(\theta)$ evaluates the scattering magnitude of the fiber in direction $\theta$. A larger $|f(\theta)|$ means the magnitude of the scattered wave in the direction $\theta$ is stronger.

Next, the process of this CP method is validated using several examples. Firstly, the single scattering by a Sic fiber embedded in the Aluminum with a pure imperfectly bonded interface $(\beta=2 \pi)$ is calculated. The stiffness of the imperfectly bonded interface is set to be $K=0.01 K_{p}$, where $K_{p}=\left(\mu_{m}+\mu_{f}\right) / 0.1 a$. The radius of the Sic fiber is chosen to be $a=71 \mu \mathrm{m}$ except if stated otherwise. Material properties of the fiber and the matrix are listed in Table 1. The comparison of the calculated $\sigma_{s}$ between the exact solutions

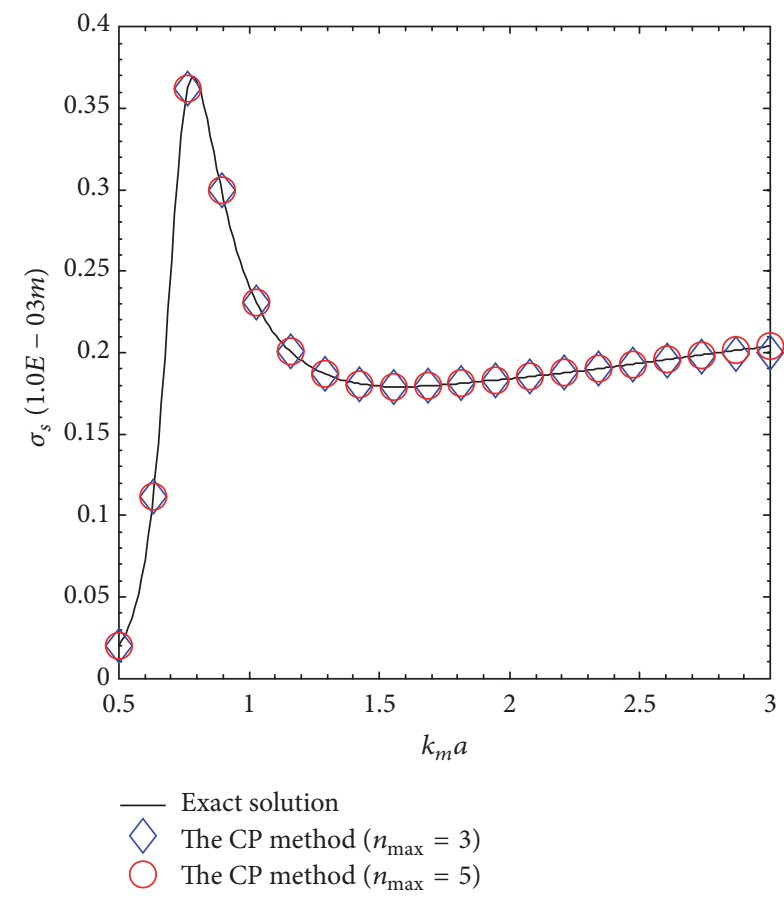

Figure 3: Comparison of $\sigma_{s}$ between the current CP method and exact solutions for the pure imperfect bonding case.

TABLE 1: Material properties of Sic and Al.

\begin{tabular}{lcc}
\hline \multirow{2}{*}{ Sic } & $\rho_{f}\left(\mathrm{~kg} / \mathrm{m}^{3}\right)$ & $\mu_{f}(\mathrm{GPa})$ \\
& 3181 & 188.1 \\
\hline \multirow{2}{*}{$\mathrm{Al}$} & $\rho_{m}\left(\mathrm{~kg} / \mathrm{m}^{3}\right)$ & $\mu_{m}(\mathrm{GPa})$ \\
& 2706 & 26.7 \\
\hline
\end{tabular}

(see the Appendix) and the current CP method at different frequencies is shown in Figure 3. For the CP method, two different $n_{\max }$ s have been considered. It is readily seen from this figure that results under these two different $n_{\max } s$ agree well with each other, only a little bit of difference exists at high frequencies, and both numerical results agree well with the exact solutions. Secondly, two single scattering problems with partial imperfectly bonded interfaces are calculated. The results are presented in Figure 4. It is clear that the results converge to the exact solutions as $n_{\max }$ increases. Additionally, comparison of the results in Figures 3 and 4 indicates that more terms in the infinite series in (1) are required for cases with partial imperfectly bonded interfaces than those with a pure imperfect and perfect bonding. The main reason may be caused by the discontinuity of displacements at the two junctions between the partial imperfect bonding and the remaining perfect bonding. In this work, $n_{\max }$ is set to be 10 in all the following calculations.

Lastly, the single scattering by a fiber with a partial debonding from the matrix is calculated. In order to model the debonding (crack), the stiffness $K$ is set to be a small value $10^{-6} K_{p}$ in the current CP method. The normalized frequency is $k_{m} a=3.0$, and the crack is located at $\alpha=0$ and $\beta=\pi$. The crack opening displacement (COD) is calculated and 


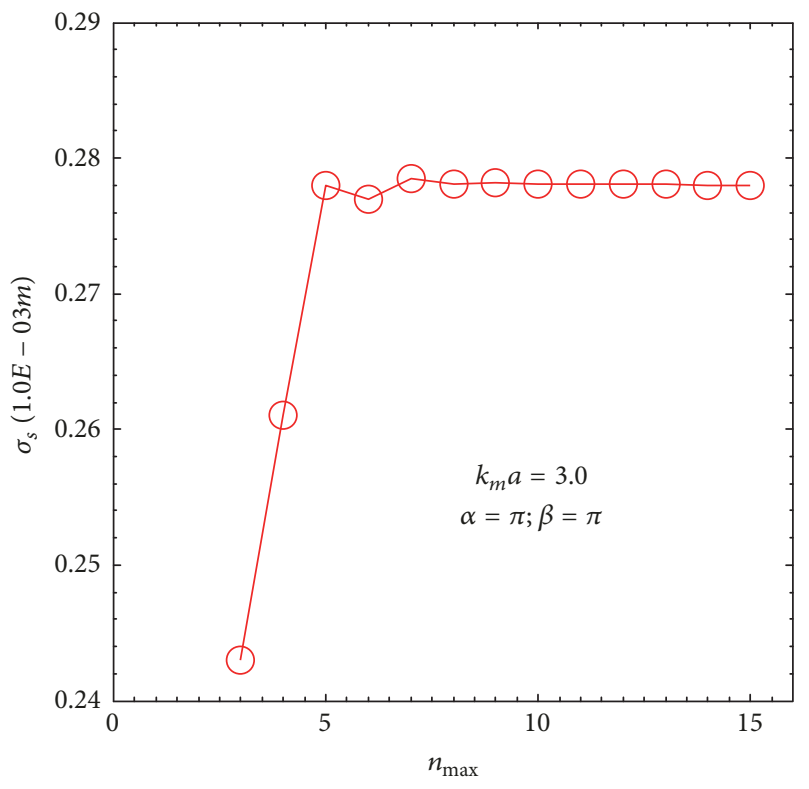

(a)

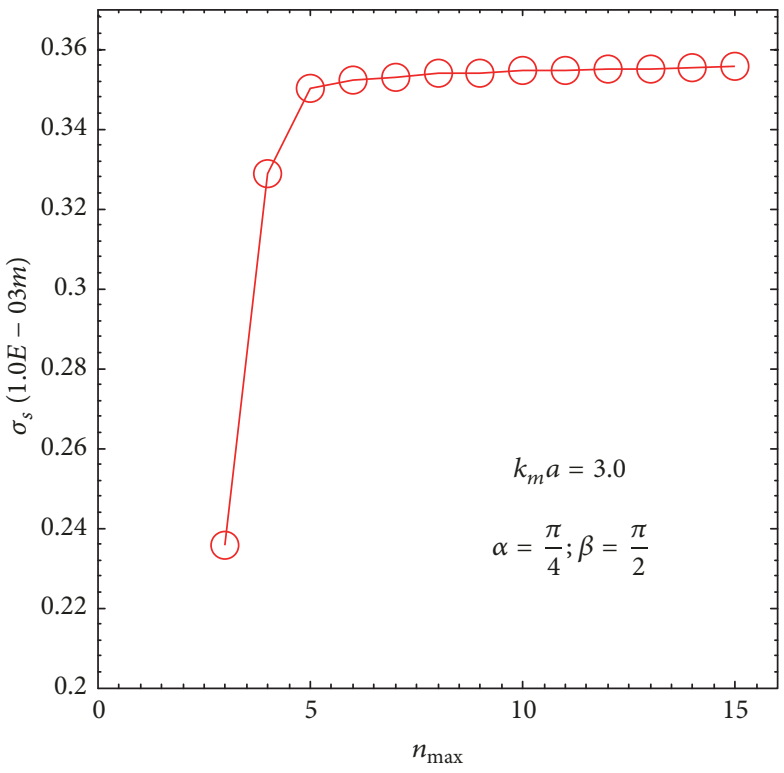

(b)

FIGURE 4: Convergence analysis of the CP method through two examples with a partial imperfectly bonded interface: (a) $\alpha=\pi, \beta=\pi$ and (b) $\alpha=\pi / 4, \beta=\pi / 2$.

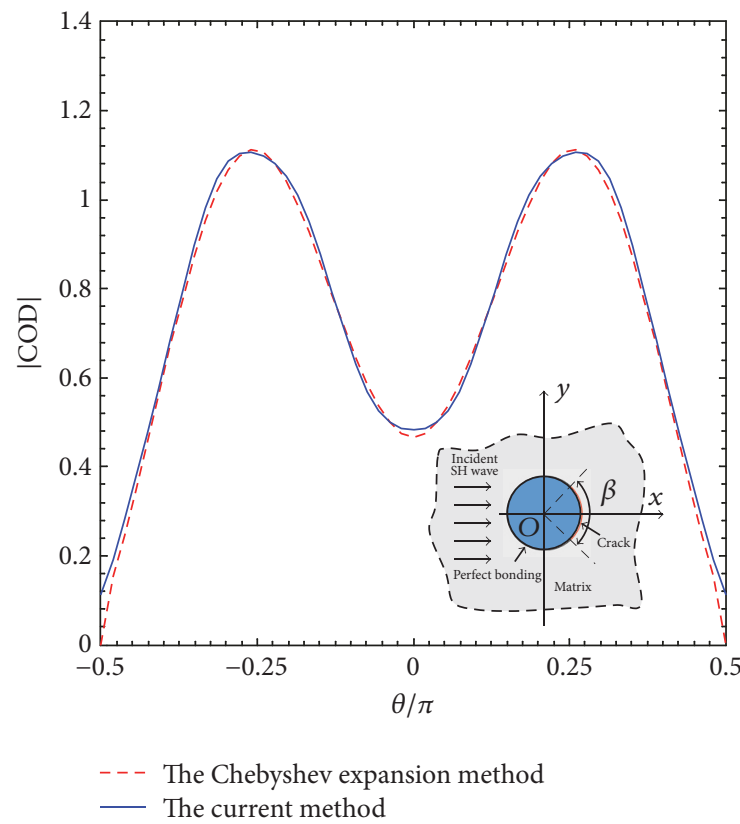

(a)

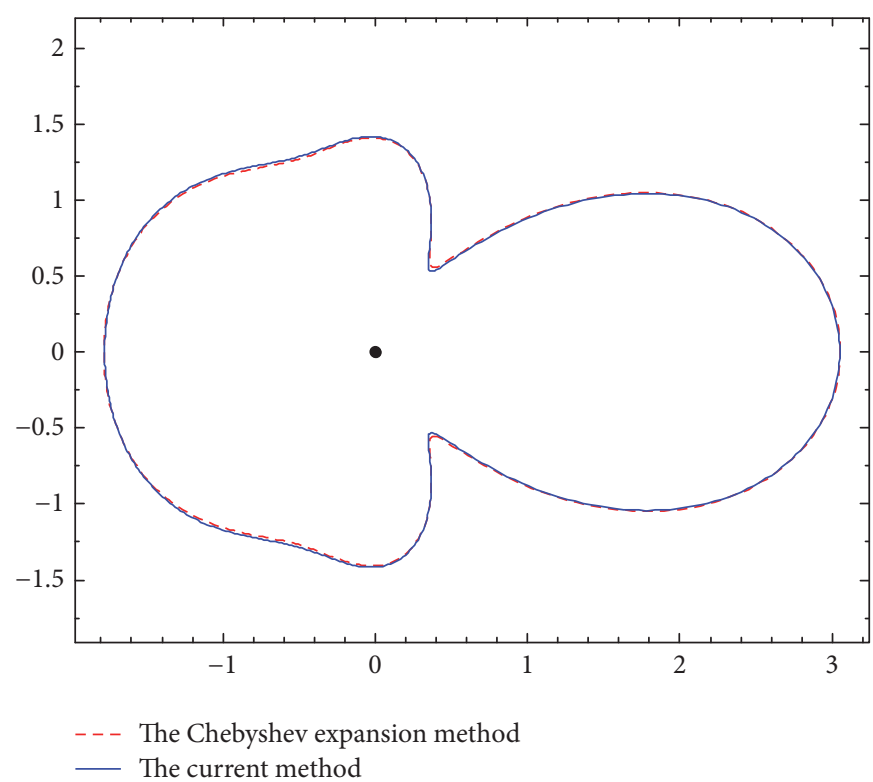

(b)

FIGURE 5: The calculated COD (a) and polar plot of the far-field scattering magnitude pattern (b) for the single scattering by a fiber with a partial debonding from the matrix.

plotted in Figure 5(a) and the far-field scattering magnitude pattern is illustrated in Figure 5(b). For a comparison, the results obtained from the Chebyshev expansion method [3638] are also illustrated in Figure 5. The results obtained from the current $\mathrm{CP}$ method are found to be in good agreement with those of the Chebyshev expansion method. All these examples show that the current $\mathrm{CP}$ method has a good performance. 


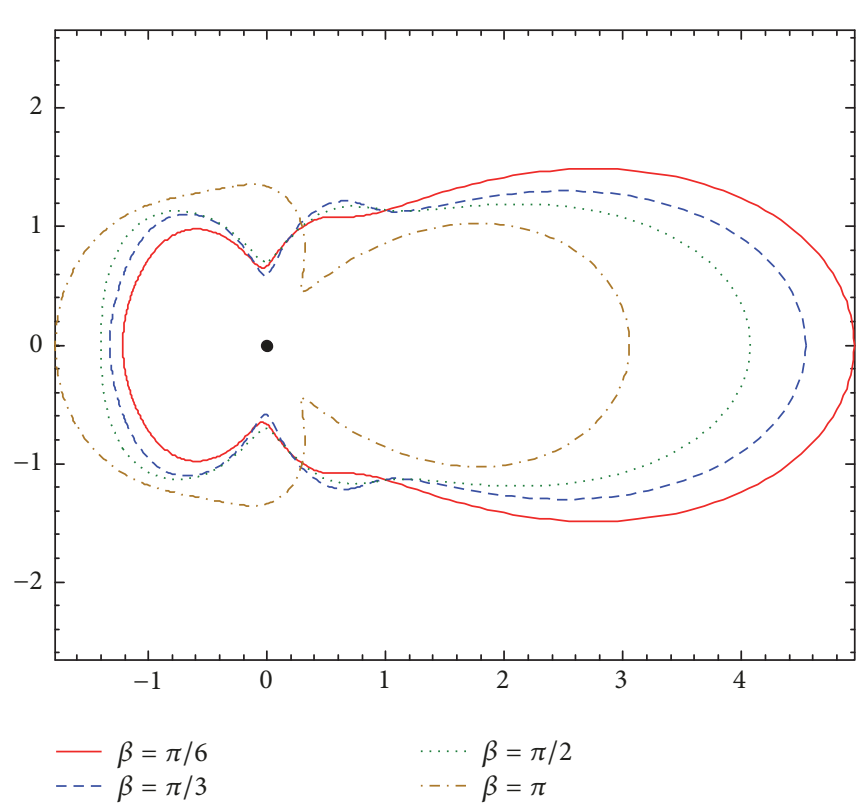

(a)

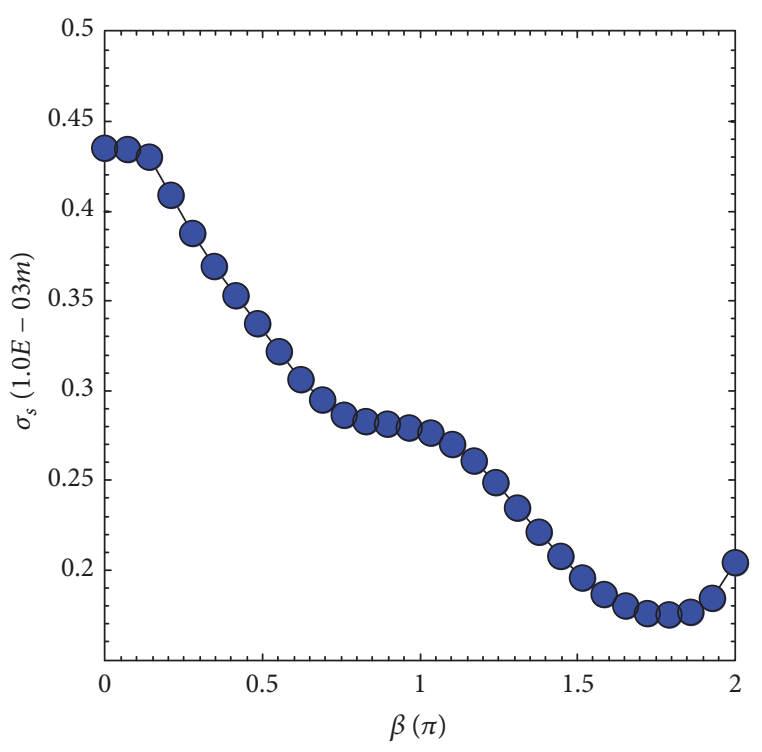

(b)

Figure 6: Influence of $\beta$ on (a) the far-field scattering magnitude and (b) $\sigma_{s}$ at $k_{m} a=3.0$ and $\alpha=0.0$.

\section{Parameter Study and Analysis}

In this section, the effects of the normalized frequency $k_{m} a$ of the incident wave, the stiffness $K$ and width, $\beta$, of the imperfectly bonded interface on the far-field scattering magnitude, scattering cross section, and scattering attenuation coefficient of coherent $\mathrm{SH}$-waves in fiber-reinforced composites are investigated.

3.1. Influence of $\beta$ on the Far-Field Scattering Magnitude and $\sigma_{s}$. In this subsection, the influence of $\beta$ on the far-field scattering magnitude and $\sigma_{s}$ is investigated. The material properties of the fiber and matrix are the same as those used before. The normalized frequency of the incident wave is $k_{m} a=3.0$. The partial imperfectly bonded interface has a stiffness $K=0.01 K_{p}$ and is located at $\alpha=0.0$. The far-field scattering magnitudes under several different $\beta$ s $(\pi / 6, \pi / 3$, $\pi / 2$ and $\pi$ ) are shown in Figure $6(\mathrm{a})$. These results show that the forward scattering (along the direction of the incident wave) decreases and the backward scattering (opposite to the direction of the incident wave) increases with the increment of $\beta . \sigma_{s}$ changing with $\beta$ is plotted in Figure $6(\mathrm{~b})$. It is clear that, in general, increment of $\beta$ reduces the scattering capacity of the fiber.

3.2. Influence of Stiffness $K$ on $\sigma_{s}$. The $\sigma_{s}$ changing with stiffness $K$ under several $\beta \mathrm{s}$ is plotted in Figure 7 . In this figure, the partial imperfectly bonded interface is also located at $\alpha=0.0$. As evident from this figure, $\sigma_{s}$ s under different $\beta$ s approach the same value as $K$ increases to a large value. The reason is that all the imperfect bonding will become the perfect one as $K$ increases. $\sigma_{s}$ under each $\beta$ approaches a constant as $K$ decreases, since the imperfect bonding will become the debonding one with the decrement of $K$. Moreover, $\sigma_{s}$

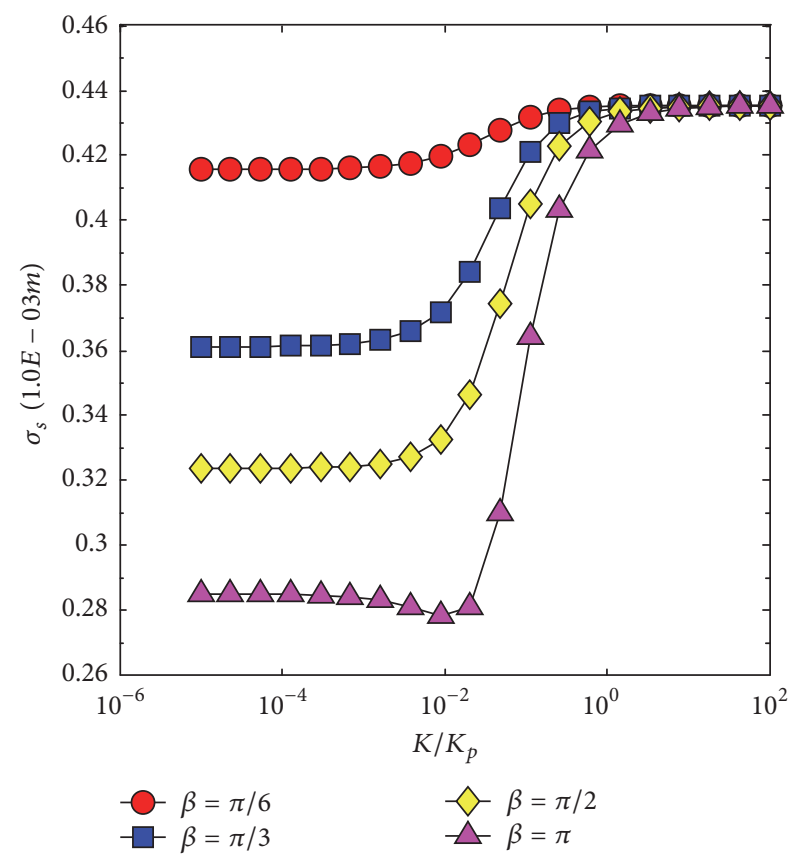

FIGURE 7: Influence of stiffness $K$ on the $\sigma_{s}$ at $k_{m} a=3.0$ under several $\beta$ s.

decreases as the imperfectly bonded interface deteriorates. With the increment of $\beta, \sigma_{s}$ becomes more sensitive to the stiffness $K$, which indicates that the ultrasonic technique is more suitable to evaluate large imperfectly bonded interfaces.

3.3. Influence of $k_{m} a$ and $\beta$ on the Scattering Attenuation of Coherent SH-Waves in Fiber-Reinforced Composites. In this work, we assume that the orientation of the partial 


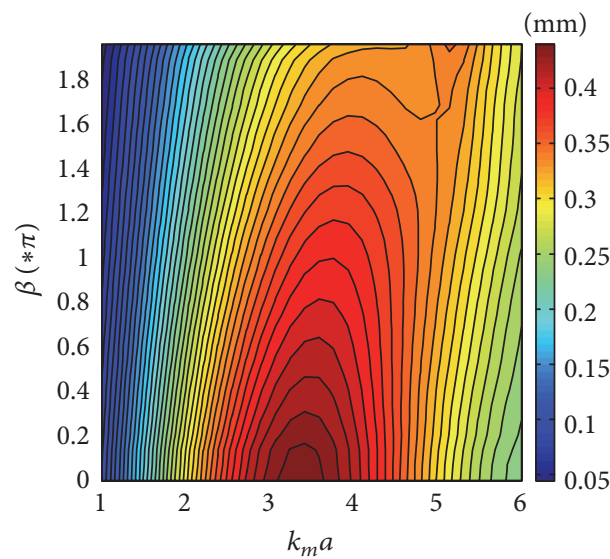

(a)

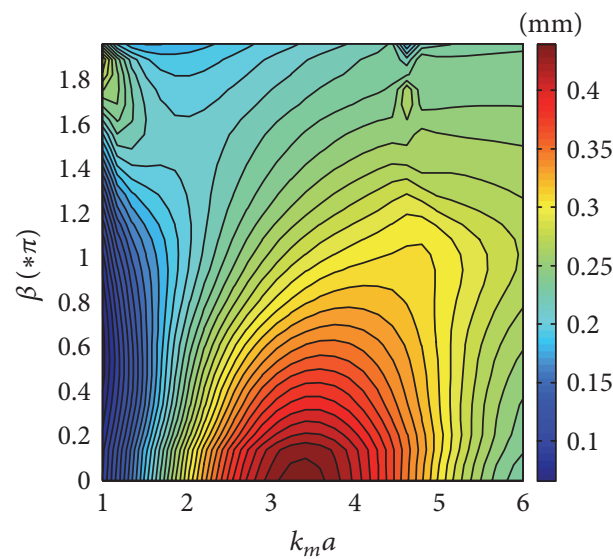

(b)

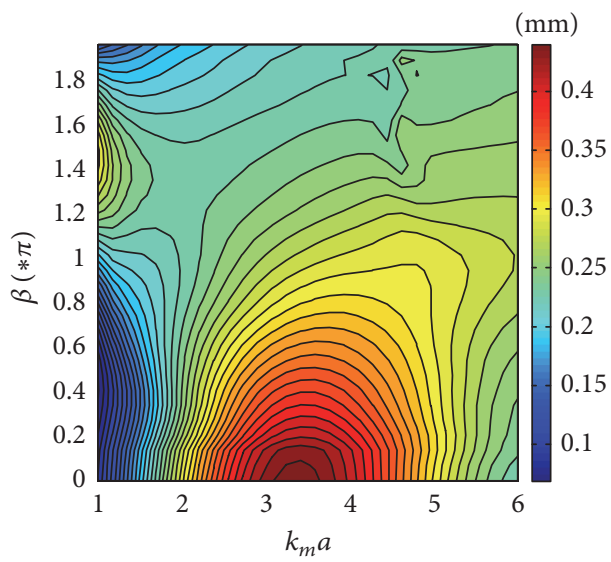

(c)

Figure 8: Contour plots of $\left\langle\sigma_{s}\right\rangle$ changing with $k_{m} a$ and $\beta$ at (a) $K / K_{p}=0.1$, (b) $K / K_{p}=0.01$, and (c) $K / K_{p}=0.001$.

imperfectly bonded interface is randomly distributed, and the width of the imperfect interface is the same for all the fibers. According to the independent scattering model, the attenuation coefficient of coherent $\mathrm{SH}$-waves in such a composite can be calculated as

$$
\alpha_{s}=\frac{1}{2}\left\langle\sigma_{s}\right\rangle n_{0}
$$

where $\left\langle\sigma_{s}\right\rangle$ is the statistical average of $\sigma_{s}$ over angle $\alpha$, with the definition as follows:

$$
\left\langle\sigma_{s}\right\rangle=\int_{0}^{2 \pi} p(\alpha) \sigma_{s}(\alpha, \beta) d \alpha,
$$

where $p(\alpha)$ is the probability density function of $\alpha$. In this work, we assume that $\alpha$ has a uniform distribution over $\left[\begin{array}{ll}0 & 2 \pi\end{array}\right]$. In the calculations, $\sigma_{s} s$ at 100 different $\alpha s$ uniformly selected within $\left[\begin{array}{ll}0 & 2 \pi\end{array}\right]$ are calculated and averaged to obtain $\left\langle\sigma_{s}\right\rangle$. The contour plots of the calculated $\left\langle\sigma_{s}\right\rangle$ changing with $k_{m} a$ and $\beta$ under three different $K s$ are shown in Figure 8 . The results indicate that $\left\langle\sigma_{s}\right\rangle$ and the scattering attenuation coefficient $\alpha_{s}$ become more sensitive to $\beta$, as the normalized frequency of the incident wave is within about $k_{m} a \in\left[\begin{array}{ll}3.0 & 4.0\end{array}\right]$. The sensitivity of $\left\langle\sigma_{s}\right\rangle$ and $\alpha_{s}$ to $\beta$ is low for both much lower and much higher frequencies of the incident wave. Accordingly, the frequency of waves should be carefully selected for the using of the ultrasonic technique to nondestructively evaluate the interfaces in composites.

\subsection{Influence of $k_{m} a$ and $K$ on the Scattering Attenuation.} In this subsection, the influence of $k_{m} a$ and the stiffness $K$ on the scattering attenuation of coherent SH-waves in fiber-reinforced composites is investigated. As before, the orientation of the partial imperfectly bonded interface is randomly distributed. The contour plots of the calculated $\left\langle\sigma_{s}\right\rangle$ changing with $k_{m} a$ and $K$ at several different $\beta$ s are shown in Figure 9. The results show that $\left\langle\sigma_{s}\right\rangle$ and the scattering attenuation are not sensitive to the initial deterioration of interfaces. As the interfaces deteriorate further, the sensitivity of $\left\langle\sigma_{s}\right\rangle$ and scattering attenuation to $K$ increases. This fact benefits the nondestructive evaluation of interfaces in composites using the scattering attenuation based ultrasonic technique. Similar to the conclusion reached in Section 3.3, the sensitivity of $\left\langle\sigma_{s}\right\rangle$ and scattering attenuation to $K$ is affected by the frequency of the incident wave. In this case, $\left\langle\sigma_{s}\right\rangle$ and the scattering attenuation become more sensitive to $K$ as the normalized frequency of the incident wave is within about $k_{m} a \in\left[\begin{array}{ll}3.0 & 4.0\end{array}\right]$. 


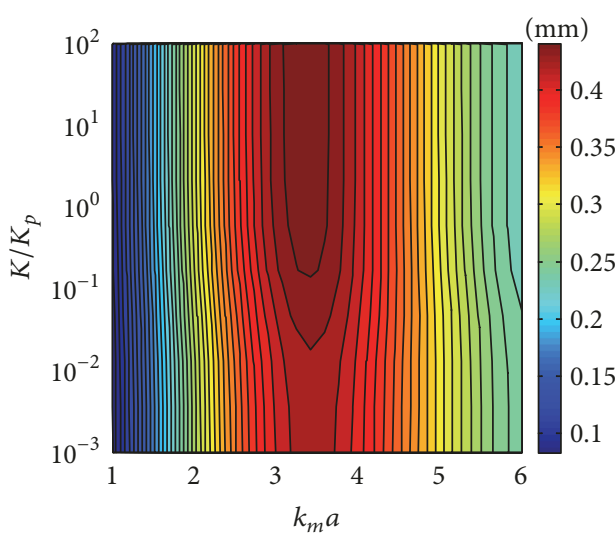

(a)

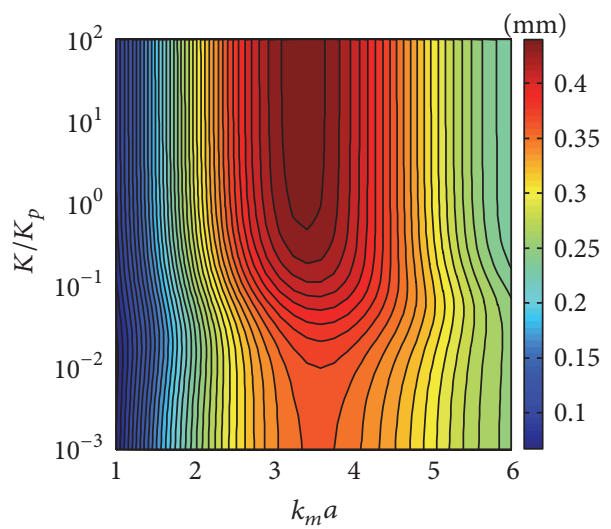

(c)

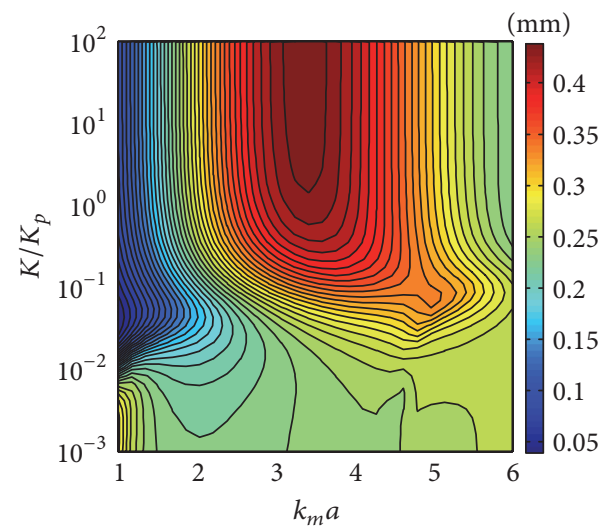

(e)

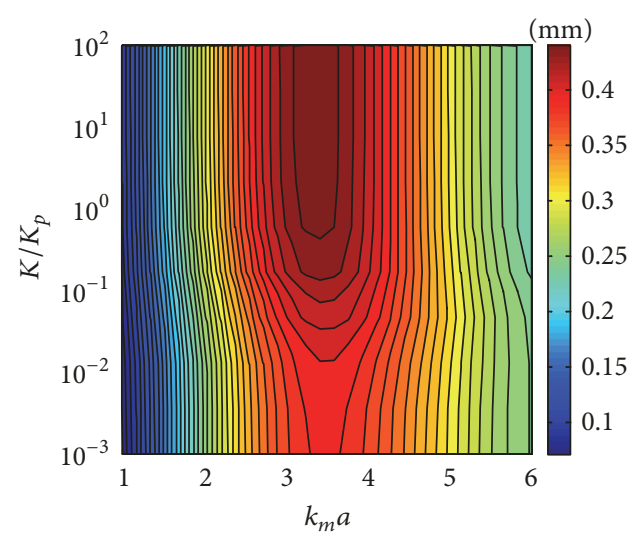

(b)

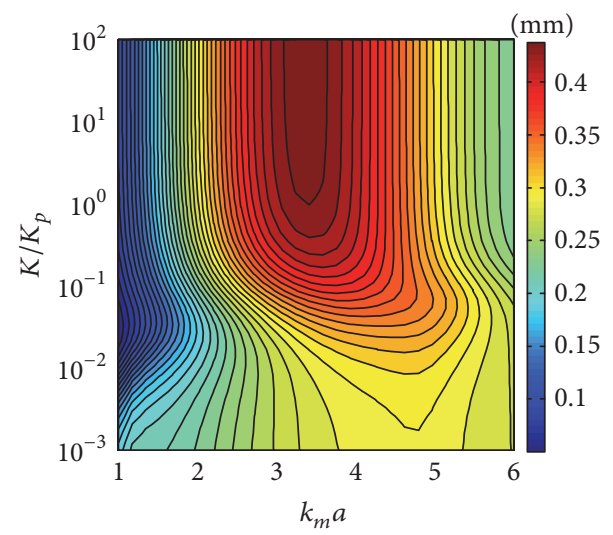

(d)

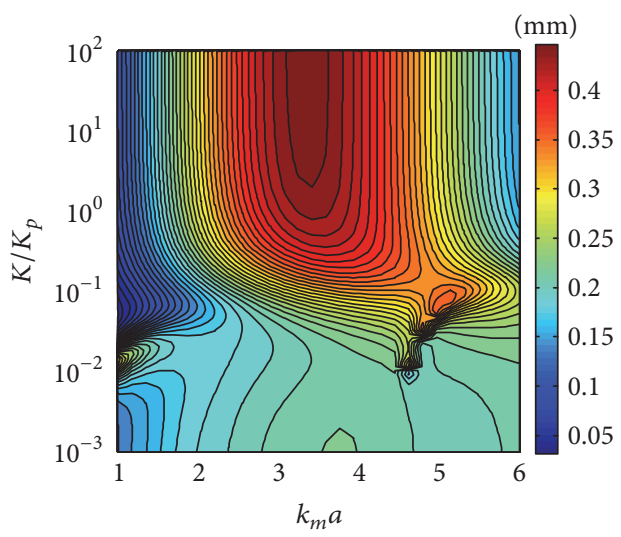

(f)

Figure 9: Contour plots of $\left\langle\sigma_{s}\right\rangle$ changing with $k_{m} a$ and $K$ at several $\beta$ s: (a) $\beta=\pi / 6$, (b) $\beta=\pi / 3$, (c) $\beta=\pi / 2$, (d) $\beta=\pi$, (e) $\beta=3 / 2 \pi$, and (f) $\beta=2 \pi$.

\section{Conclusions}

The influence of the partial imperfectly bonded interfaces between each fiber and the matrix on the far-field scattering magnitude, scattering cross section, and scattering attenuation of coherent $\mathrm{SH}$-waves in fiber-reinforced composites is investigated. The imperfection of interfaces is modelled using the spring model, and the CP method is proposed to solve the single scattering problem with such a partial imperfectly bonded interface. Based on the analysis of the single scattering problems, the effects of frequency of the incident wave and parameters of the partial imperfectly bonded interfaces on the far-field scattering magnitude, scattering cross section, and the scattering attenuation of coherent $\mathrm{SH}$-waves in fiber-reinforced composites are investigated numerically. The main contribution of this work includes the following:

(i) The CP method is proposed to solve the single scattering of SH-waves with a partial imperfectly bonded interface between the fiber and the matrix. Compared 


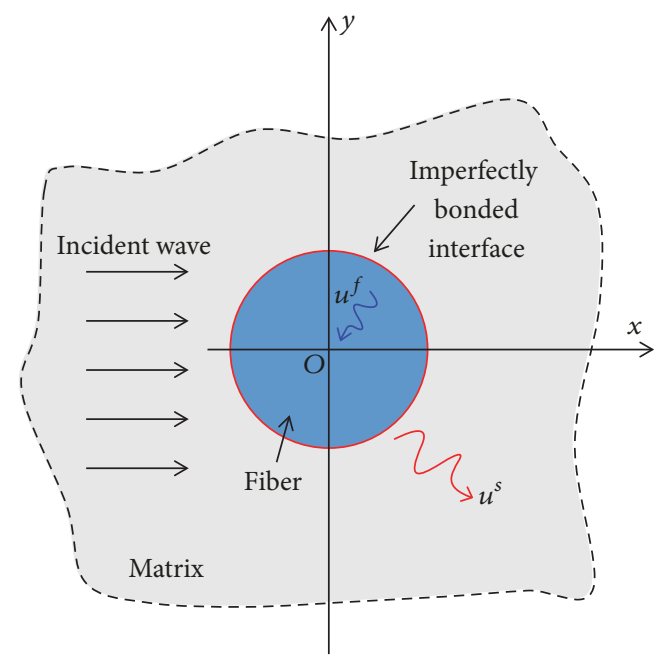

FIGURE 10: Scattering of a plane SH-wave by a cylindrical fiber with a pure imperfectly bonded interface.

with other existing techniques, the derivation process and implementation of the current $\mathrm{CP}$ method are simple and it has a comparable accuracy. The disadvantage is that it cannot give accurate solutions at points near the two tips of the imperfect interface. In general, the current CP method can be taken as an auxiliary one for the existing methods.

(ii) Extensive parameter study shows that the sensitivity of scattering attenuation of coherent $\mathrm{SH}$ waves in fiber-reinforced composites to the imperfection/deterioration of interfaces is significantly affected by the frequency of the incident wave. Therefore, waves should be carefully selected for the use of the scattering attenuation based ultrasonic technique to nondestructively evaluate the interfaces in composites.

(iii) Initial deterioration of the interfaces is relatively difficult to be detected using the scattering attenuation based ultrasonic technique.

Although this work only considers the SH-waves, the analysis framework can be extended to more complicated cases, such as the P- and SV-waves, without significant difficulties.

\section{Appendix}

\section{Exact Solutions of the Single Scattering Problem with a Pure Imperfectly Bonded Interface}

Figure 10 shows the schematic of the single scattering of a plane $\mathrm{SH}$-wave with a unit magnitude by a fiber with a pure imperfectly bonded interface to the matrix. $u^{s}$ is the scattered wave and $u^{f}$ is the refracted wave into the fiber. The incident, scattered, and refracted waves can be expressed in series as those in (1).

In this case, the two boundary conditions of displacements and stresses are

$$
\begin{gathered}
\left.\sigma_{r z}^{+}\right|_{r=a}=\left.\sigma_{r z}^{-}\right|_{r=a}, \quad \theta \in\left[\begin{array}{ll}
0 & 2 \pi
\end{array}\right], \\
\left.u^{+}\right|_{r=a}-\left.u^{-}\right|_{r=a}=\left.\frac{1}{K} \sigma_{r z}^{+}\right|_{r=a}, \quad \theta \in\left[\begin{array}{ll}
0 & 2 \pi
\end{array}\right] .
\end{gathered}
$$

Since $\theta$ varies from 0 to $2 \pi$ in the above equation, the orthogonality of trigonometric functions is available. Then substitution of the displacements in (1) and the corresponding expressions for the stresses into (A.1) yields the analytical solutions for $A_{n}$ and $B_{n}$ :

$$
\begin{aligned}
& A_{n}=-\mathbf{i}^{n} \frac{J_{n}\left(k_{m} a\right)-\left[\gamma\left(J_{n}\left(k_{f} a\right) / J_{n}^{\prime}\left(k_{f} a\right)\right)+\mu_{m} k_{m} / K\right] J_{n}^{\prime}\left(k_{m} a\right)}{H_{n}^{(1)}\left(k_{m} a\right)-\left[\gamma\left(J_{n}\left(k_{f} a\right) / J_{n}^{\prime}\left(k_{f} a\right)\right)+\mu_{m} k_{m} / K\right] H_{n}^{(1)^{\prime}}\left(k_{m} a\right)}, \quad n=-\infty, \ldots, \infty, \\
& B_{n}=\gamma \frac{\mathbf{i}^{n} J_{n}^{\prime}\left(k_{m} a\right)+A_{n} H_{n}^{(1)^{\prime}}\left(k_{m} a\right)}{J_{n}^{\prime}\left(k_{f} a\right)} .
\end{aligned}
$$

\section{Disclosure}

Part of this work has been presented orally at "The 2nd International Conference on Computational Engineering and Science for Safety and Environmental Problems," 2017.

\section{Conflicts of Interest}

The authors declare that they have no conflicts of interest.

\section{Acknowledgments}

This work was supported by the Chinese National Natural Science Fund (Grants 11502036, 11372104, and 11632004) and the Natural Science Fund of the city of Chongqing (Grant cstc2015jcyjA0577). The Key Program for International Science and Technology Cooperation Projects of the Ministry of Science and Technology of China (no. 2016YFE0125900) and the Guangxi Key Laboratory of Manufacturing Systems and Advanced Manufacturing Technology (no. 16-380-12-014k) also provided partial financial support.

\section{References}

[1] S. I. Rokhlin, W. Huang, and Y. C. Chu, "Ultrasonic scattering and velocity methods for characterization of fibre-matrix interphases," Ultrasonics, vol. 33, no. 5, pp. 351-364, 1995. 
[2] R.-B. Yang and A. K. Mal, "Elastic waves in a composite containing inhomogeneous fibers," International Journal of Engineering Science, vol. 34, no. 1, pp. 67-79, 1996.

[3] Y.-S. Wang and D. Wang, "Scattering of elastic waves by a rigid cylindrical inclusion partially debonded from its surrounding matrix - I. SH case," International Journal of Solids and Structures, vol. 33, no. 19, pp. 2789-2815, 1996.

[4] X. Ji, X. Luo, Y. Q. Yang, J. H. Lou, B. Huang, and X. R. Wang, "Research progress of nondestructive testing for continuous fiber-reinforced metal-matrix composites," Rare Metal Materials and Engineering, vol. 42, pp. 401-405, 2013.

[5] L. L. Shaw, P. Karpur, and T. E. Matikas, "Fracture strength and damage progression of the fiber/matrix interfaces in titaniumbased MMCs with different interfacial layers," Composites Part B: Engineering, vol. 29, no. 3, pp. 331-339, 1998.

[6] Y.-S. Wang and D. Wang, "Scattering of elastic waves by a rigid cylindrical inclusion partially debonded from its surrounding matrix - II. P and SV cases," International Journal of Solids and Structures, vol. 33, no. 19, pp. 2817-2840, 1996.

[7] T. H. Ju and S. K. Datta, "Dynamics of a laminated composite plate with interface layers," Journal of Nondestructive Evaluation, vol. 11, no. 3-4, pp. 227-235, 1992.

[8] L. L. Foldy, "The multiple scattering of waves. I. General theory of isotropic scattering by randomly distributed scatterers," Physical Review A: Atomic, Molecular and Optical Physics, vol. 67, no. 3-4, pp. 107-119, 1945.

[9] S. Biwa, S. Idekoba, and N. Ohno, "Wave attenuation in particulate polymer composites: Independent scattering/absorption analysis and comparison to measurements," Mechanics of Materials, vol. 34, no. 10, pp. 671-682, 2002.

[10] J. Zhang and W. Ye, "An explicit formula for the coherent SH waves' attenuation coefficient in random porous materials with low porosities," Ultrasonics, vol. 62, pp. 27-34, 2015.

[11] S. K. Kanaun and V. M. Levin, "Effective medium method in the problem of axial elastic shear wave propagation through fiber composites," International Journal of Solids and Structures, vol. 40, no. 18, pp. 4859-4878, 2003.

[12] P. G. Bussink, P. L. Iske, J. Oortwijn, and G. L. Verbist, "Selfconsistent analysis of elastic wave propagation in two-dimensional matrix-inclusion composites," Journal of the Mechanics and Physics of Solids, vol. 43, no. 10, pp. 1673-1690, 1995.

[13] J.-Y. Kim, J.-G. Ih, and B.-H. Lee, "Dispersion of elastic waves in random particulate composites," The Journal of the Acoustical Society of America, vol. 97, no. 3, pp. 1380-1388, 1995.

[14] S. K. Bose and A. K. Mal, "Axial shear waves in a medium with randomly distributed cylinders," The Journal of the Acoustical Society of America, vol. 55, no. 3, pp. 519-523, 1974.

[15] V. K. Varadan, Y. Ma, and V. V. Varadan, "A multiple scattering theory for elastic wave propagation in discrete random media," The Journal of the Acoustical Society of America, vol. 77, no. 2, pp. 375-385, 1985.

[16] V. K. Varadan, V. V. Varadan, and Y. . Pao, "Multiple scattering of elastic waves by cylinders of arbitrary cross section. I. SH waves," The Journal of the Acoustical Society of America, vol. 63, no. 5, pp. 1310-1319, 1978.

[17] A. I. Beltzer, "The effective dynamic response of random composites and polycrystals-a survey of the causal approach," Wave Motion, vol. 11, no. 3, pp. 211-229, 1989.

[18] A. I. Beltzer and N. Brauner, "The dynamic response of random composites by a causal differential method," Mechanics of Materials, vol. 6, no. 4, pp. 337-345, 1987.
[19] S. Biwa, "Independent scattering and wave attenuation in viscoelastic composites," Mechanics of Materials, vol. 33, no. 11, pp. 635-647, 2001.

[20] A. N. Norris and J.-M. Conoir, "Multiple scattering by cylinders immersed in fluid: High order approximations for the effective wavenumbers," The Journal of the Acoustical Society of America, vol. 129, no. 1, pp. 104-113, 2011.

[21] N. Brauner and A. I. Beltzer, "Wave-obstacle interaction in a lossy medium: energy perturbations and negative extinction," Ultrasonics, vol. 26, no. 6, pp. 328-334, 1988.

[22] C. C. Mow and Y. H. Pao, The diffraction of elastic waves and dynamic stress concentrations, 1971.

[23] N. M. Newmark, "Tests and analysis of composite beams with incomplete interaction," in Proceedings of the Society for Experimental Stress Analysis, vol. 9, pp. 75-92, 1951.

[24] J.-M. Baik and R. B. Thompson, "Ultrasonic scattering from imperfect interfaces: A quasi-static model," Journal of Nondestructive Evaluation, vol. 4, no. 3-4, pp. 177-196, 1984.

[25] W. Huang, S. Brisuda, and S. I. Rokhlin, "Ultrasonic wave scattering from fiber-matrix interphases," The Journal of the Acoustical Society of America, vol. 97, no. 2, pp. 807-817, 1995.

[26] P. A. Martin, "Boundary integral equations for the scattering of elastic waves by elastic inclusions with thin interface layers," Journal of Nondestructive Evaluation, vol. 11, no. 3-4, pp. 167-174, 1992.

[27] J. A. Otero, R. Rodriguez-Ramos, G. Monsivais, C. Stern, R. Martínez, and R. Dario, "Interfacial waves between two magneto-electro-elastic half-spaces with magneto-electro-mechanical imperfect interface," Philosophical Magazine Letters, vol. 94, no. 10, pp. 629-638, 2014.

[28] H. Brito-Santana, R. de Medeiros, R. Rodriguez-Ramos, and V. Tita, "Different interface models for calculating the effective properties in piezoelectric composite materials with imperfect fiber-matrix adhesion," Composite Structures, vol. 151, pp. 7080, 2016.

[29] J. Aboudi, "Damage in composites-Modeling of imperfect bonding," Composites Science and Technology, vol. 28, no. 2, pp. 103-128, 1987.

[30] A. K. Mal and S. K. Bose, "Dynamic elastic moduli of a suspension of imperfectly bonded spheres," in Proceedings of the, vol. 76, pp. 587-600.

[31] J. C. Lopez-Realpozo, R. Rodriguez-Ramos, R. Guinovart-Diaz et al., "Effective elastic shear stiffness of a periodic fibrous composite with non-uniform imperfect contact between the matrix and the fibers," International Journal of Solids and Structures, vol. 51, no. 6, pp. 1253-1262, 2014.

[32] M. Wurkner, H. Berger, and U. Gabbert, "Numerical investigations of effective properties of fiber reinforced composites with parallelogram arrangements and imperfect interface," Composite Structures, vol. 116, no. 1, pp. 388-394, 2014.

[33] E. Vilchevskaya and I. Sevostianov, "Overall thermal conductivity of a fiber reinforced composite with partially debonded inhomogeneities," International Journal of Engineering Science, vol. 98, pp. 99-109, 2016.

[34] S. Biwa and T. Shibata, "Elastic and ultrasonic properties of a unidirectional composite with partially debonded fibres: Numerical analysis for longitudinal shear modes," Composites Science and Technology, vol. 60, no. 1, pp. 83-93, 2000.

[35] H. Sato and Y. Shindo, "Influence of microstructure on scattering of plane elastic waves by a distribution of partially debonded elliptical inclusions," Mechanics of Materials, vol. 34, no. 7, pp. 401-409, 2002. 
[36] Y. Yang and A. Norris, "Longitudinal wave scattering from a partially bonded fiber," Wave Motion, vol. 15, no. 1, pp. 43-59, 1992.

[37] J.-Y. Kim, "Attenuation and speed of antiplane shear wave in fiber-reinforced composites with random interfacial cracks," International Journal of Solids and Structures, vol. 38, no. 40-41, pp. 7121-7137, 2001.

[38] W. Liu and R. D. Kriz, "Shear waves in fiber-reinforced composites with interfacial cracks," International Journal of Solids and Structures, vol. 35, no. 13, pp. 1425-1449, 1998.

[39] S. Biwa, S. Yamamoto, F. Kobayashi, and N. Ohno, "Computational multiple scattering analysis for shear wave propagation in unidirectional composites," International Journal of Solids and Structures, vol. 41, no. 2, pp. 435-457, 2004.

[40] J. Zhang, W. Ye, and T. X. Yu, "Numerical simulation of effective phase velocity and attenuation of shear elastic wave propagation in unidirectional composite materials," Ultrasonics, vol. 53, no. 6, pp. 1200-1211, 2013. 


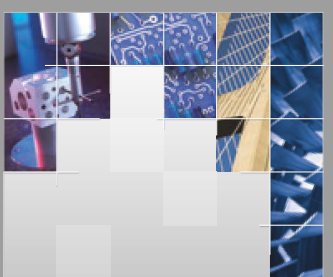

\section{Enfincering}
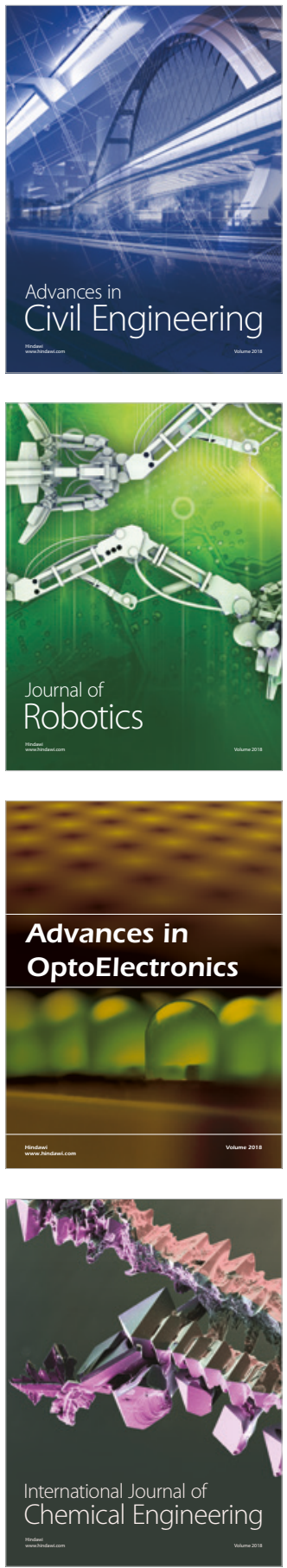

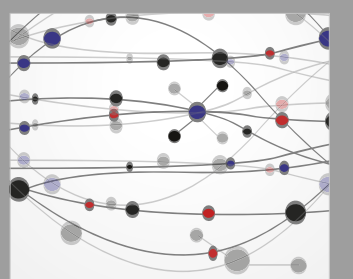

\section{Rotating \\ Machinery}

The Scientific World Journal

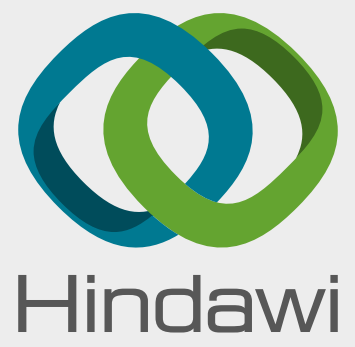

Submit your manuscripts at

www.hindawi.com
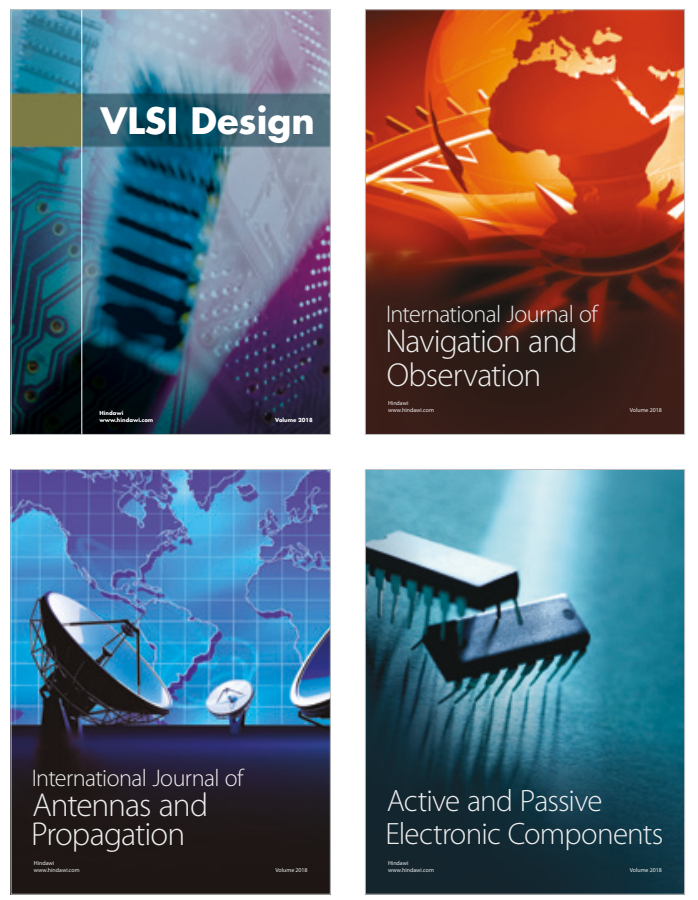
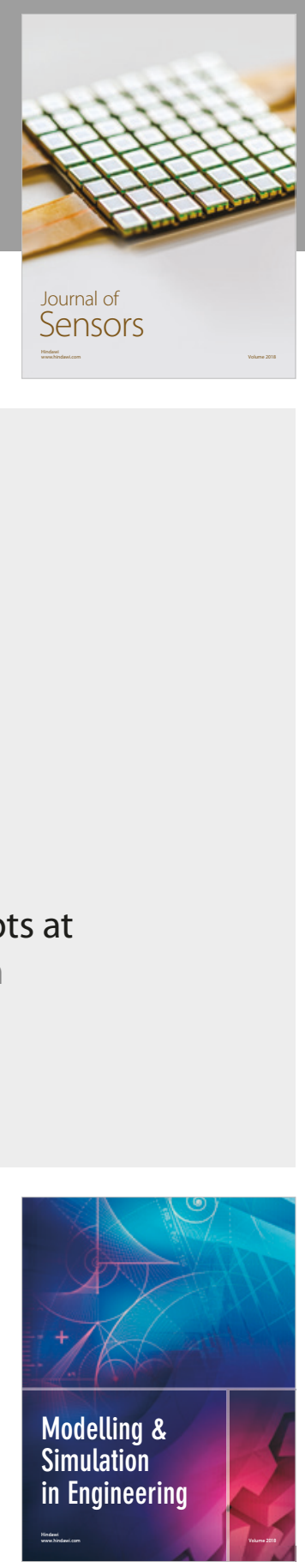

\section{Advances \\ Multimedia}
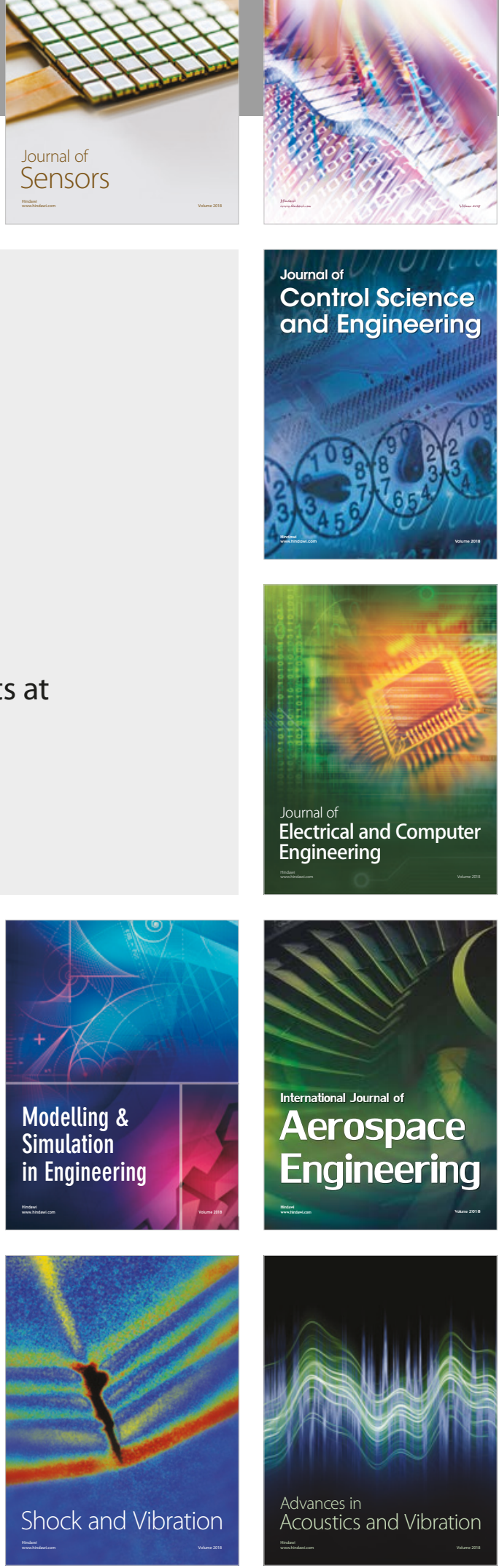